\title{
Improved Performance of an Epoxy Matrix as a Result of Combining Graphene Oxide and Reduced Graphene
}

\author{
L. Ramos-Galicia, ${ }^{1}$ L. N. Mendez, ${ }^{1}$ Ana Laura Martínez-Hernández, ${ }^{2,3}$ \\ A. Espindola-Gonzalez, ${ }^{1}$ I. R. Galindo-Esquivel, ${ }^{1}$ R. Fuentes-Ramirez, ${ }^{1}$ \\ and C. Velasco-Santos ${ }^{2,3}$ \\ ${ }^{1}$ División de Ciencias Naturales y Exactas, Facultad de Química, Universidad de Guanajuato, Noria Alta s/n Col. Noria Alta, \\ CP 36050 Guanajuato, GTO, Mexico \\ ${ }^{2}$ Centro de Física Aplicada y Tecnología Avanzada, Universidad Nacional Autónoma de México, Boulevard Juriquilla 3001, \\ Campus Juriquilla, CP 76230 Querétaro, QRO, Mexico \\ ${ }^{3}$ División de Estudios de Posgrado e Investigación, Instituto Tecnológico de Querétaro, Avenida Tecnológico s/n \\ Esq. Gral. Mariano Escobedo, Col. Centro Histórico, CP 76000 Querétaro, QRO, Mexico
}

Correspondence should be addressed to C. Velasco-Santos; cylaura@gmail.com

Received 26 September 2013; Revised 14 November 2013; Accepted 14 November 2013

Academic Editor: Giridhar Madras

Copyright (C) 2013 L. Ramos-Galicia et al. This is an open access article distributed under the Creative Commons Attribution License, which permits unrestricted use, distribution, and reproduction in any medium, provided the original work is properly cited.

We present an easy and effective way to improve the mechanical properties of an epoxy matrix by reinforcing it with a combination of graphene oxide (GO) and reduced graphene oxide (RGO). These nanocomposites were prepared with different load of nanofillers: $0.1,0.4,0.7,1.0 \mathrm{wt} \%$ and a neat epoxy. Ratios of graphene oxide and reduced graphene (GO:RGO) employed were: $0: 1,0.25: 0.75$, $0.5: 0.5,0.75: 0.25$, and $1: 0$. Results show that with only $0.4 \mathrm{wt} \%$ and a ratio $0.2: 0.75$ of GO:RGO, tensile strength and tensile toughness are $52 \%$ and $152 \%$ higher than neat epoxy while modulus of elasticity was improved $\sim 20 \%$. The obtained results suggest that it is possible achieve advantageous properties by combining graphene in oxidized and reduced conditions as it shows a synergic effect by the presence of both nanofillers.

\section{Introduction}

Graphene is a single two-dimensional layer of carbon atoms bound in a hexagonal lattice structure. This material has been extensively studied since it was first isolated in 2004 [1]. In recent years, several works have been published on the topic of graphene, discussing its extraordinary mechanical, electronic, and thermal properties [2-5]. Cooper et al. [6] presented an extensive review of the most important experimental work on novel applications of graphene, and a section was dedicated to discuss its amazing mechanical properties. In other works, graphene has been mixed with carbon nanotubes in order to enhance the performance of the reinforced matrix. Song et al. [7] found multiple synergies in a polypropylene matrix using reduced graphene oxide and carbon nanotubes polymer latex coated. Chatterjee et al. [8] studied the reinforcement of an epoxy matrix with the addition of various mixture ratios of carbon nanotubes with graphene nanoplatelets and founding synergistic effects especially for the flexural modulus. However, it is possible to achieve synergy effects employing the same nanomaterial with different surface polarity, as this research proposes, in this case, graphene oxide and reduced graphene oxide. Young et al. [9] published a review concerning graphene and graphene oxide nanocomposites evaluating these materials' properties. They comment that graphene oxide has some advantages over graphene, such as facility to obtain it in large quantities, presence of functional groups for bonding with a polymer matrix, easy to exfoliate and disperse it, in water or a polar solvent, and hence, can be readily mixed with water-soluble polymers [10]. Graphene oxide increases the mechanical properties of nanocomposites. Bortz et al. [11] found for an epoxy resin that the fracture energy $G_{\text {IC }}$ was more than doubled and the fatigue resistance increased 


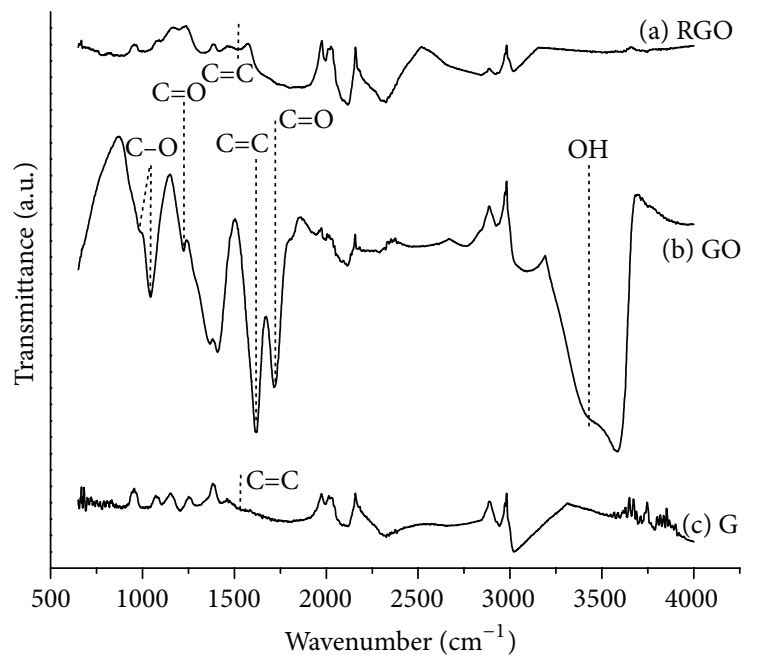

Figure 1: Infrared spectra of (a) reduced graphene (RGO), (b) graphene oxide $(\mathrm{GO})$, and (c) pure graphite $(\mathrm{G})$.

by several orders of magnitude. But some authors have encountered that functional groups over similar nanofillers alter the stoichiometric ratio [12]. On the other hand, reduced graphene has less defects on its structure [13] and does not form chemical bonds with the matrix, but, due to its hydrophobic character, it is more compatible with some polymers such as the epoxy resin, as it is observed in this study. Then, in this work it is proposed to use different graphene oxide and reduced graphene mixtures as reinforcement to enhance compatibility with an epoxy matrix keeping enough oxygen groups to bind to the polymer.

\section{Materials and Methods}

2.1. Materials. The polymeric matrix used was an epoxy resin. Diglycidyl ether of bisphenol A (DGEBA, D-3415) with a molecular weight of $340.41 \mathrm{~g} / \mathrm{mol}$ and ethylenediamine (EDA, molecular weight of $60.10 \mathrm{~g} / \mathrm{mol}$ ) were provided by Sigma-Aldrich and BAKER, respectively. Crystalline graphite, Electron Microscope Science (no. 70230), was used to prepare graphene oxide. Ascorbic acid (L-AA), supplied by Reasol, was the reducing agent for obtaining reduced graphene oxide.

2.2. Graphene Oxide and Reduced Graphene Preparation. The Hummers method was employed to prepare graphite oxide. $2 \mathrm{~g}$ of graphite powder and $46 \mathrm{~mL}$ of $\mathrm{H}_{2} \mathrm{SO}_{4}$ were introduced into a ball flask while maintaining temperature of $0^{\circ} \mathrm{C}$, and $6 \mathrm{~g}$ of $\mathrm{KMnO}_{4}$ were added slowly. This oxidation process was carried out for 2 hours at $35^{\circ} \mathrm{C}\left( \pm 2^{\circ}\right)$, under magnetic stirring. After this time, $10 \mathrm{~mL}$ of $30 \% \mathrm{H}_{2} \mathrm{O}_{2}$ and excess water were added. The resulting mixture was filtered and washed until a neutral $\mathrm{pH}$ was achieved. This product was dried at $65^{\circ} \mathrm{C}$ for 12 hours to obtain graphite oxide. Next, $100 \mathrm{mg}$ of dried graphite oxide was dispersed in $10 \mathrm{~mL}$ of water and this solution was sonicated for 3 hours in an ultrasound bath at a frequency of $50-60 \mathrm{~Hz}$, and then graphene oxide was obtained. A portion of graphene oxide was dried to be employed as a reinforcement powder. The another portion of the sonicated solution was heated to $95^{\circ} \mathrm{C}$ and added ascorbic acid $(2 \mathrm{mM})$ reacting for 15 minutes [14]. Finally, reduced graphene was washed and dried at $65^{\circ} \mathrm{C}$ for 12 hours.

2.3. Preparation of Epoxy Nanocomposites. Nanocomposites were prepared at different load of nanofillers: $0.1,0.4,0.7$, and $1.0 \mathrm{wt} \%$ and each load was mixed in different ratios of graphene oxide and reduced graphene (GO: RGO): $0: 1$, $0.25: 0.75,0.5: 0.5,0.75: 0.25$, and $1: 0$. Nanofillers were added to DGEBA then sonicated during 1 hour to improve the dispersion. Then, ethylenediamine was incorporated to empty this mix in Teflon mould with dimensions according to norm ASTM D6338 for determining mechanical properties. These samples were cured at $50^{\circ} \mathrm{C}$ for 1 hour. In addition, a neat epoxy was prepared for comparison purpose.

2.4. Characterization of Graphene Oxide and Reduced Graphene. Infrared (IR) spectroscopy, transmission electron microscopy (TEM), and energy-dispersive X-ray spectroscopy (EDS) were used as characterization techniques for both nanomaterials. Infrared spectra were obtained on transmittance mode by a FT-IR Spectrum 100 PerkinElmer spectrometer. TEM micrographs were obtained through a JEOL TEM-1010 microscope operating at $80 \mathrm{kV}$. EDS was adapted in equipment JEOL JSM-6060 LV scanning electron microscopy. In addition, dispersion tests were performed adding $2 \mathrm{mg}$ of GO and RGO in $10 \mathrm{~mL}$ of water, DGEBA, and hexane. They were sonicated during 10 minutes and their dispersion was observed at different times.

2.5. Characterization of Nanocomposites. Tensile tests were carried out using an AGS-J SHIMADZU universal tester at $15 \mathrm{~mm} / \mathrm{min}$. Dog-bone sample dimensions were according to norm ASTM D-638.

\section{Results and Discussion}

3.1. Characterization of Nanofillers. Figure 1 shows infrared spectra of (a) crystalline graphite, (b) graphene oxide, and (c) reduced graphene. An inactive band for pure graphite can be observed; at $\sim 1500-1600 \mathrm{~cm}^{-1}$ in-plane stretching vibration of $\mathrm{sp}^{2}$-hybridized $\mathrm{C}=\mathrm{C}$ is found [15]. New bands appear for graphene oxide such as a signal at $3162 \mathrm{~cm}^{-1}$ corresponding to $\mathrm{OH}$ groups. At $1710 \mathrm{~cm}^{-1}$, the stretching vibration of $\mathrm{C}=\mathrm{O}$ related to carboxyl groups is found, whereas at $1220 \mathrm{~cm}^{-1}$ and $1050 \mathrm{~cm}^{-1}$, the presence of epoxy and alkoxy groups is observed, respectively. These bands corroborate an oxidation process with different groups that can bond to the matrix polymer. After the reduction step using L-AA, most of the oxygen groups disappeared and the resulting spectrum tends to be like crystalline graphite spectrum, even though a few oxygen groups remain over the nanosheets.

Figure 2 exhibits TEM morphology of the graphene oxide (a-b) and reduced graphene (c-d). As it is observed, chemical process applied in this work produces few layers with 


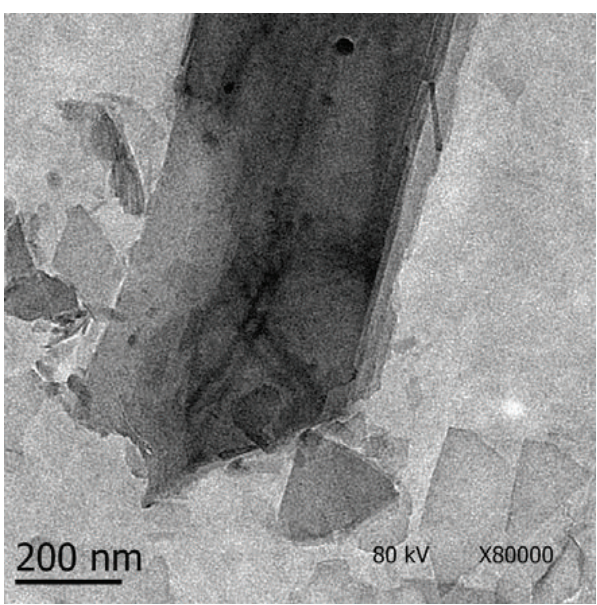

(a)

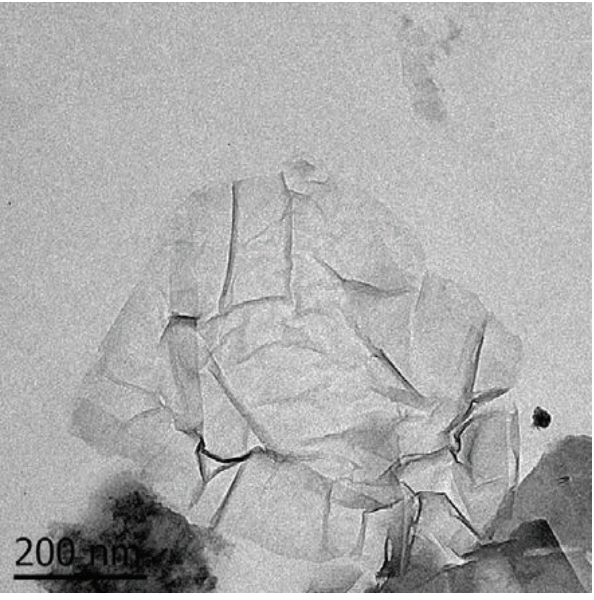

(c)

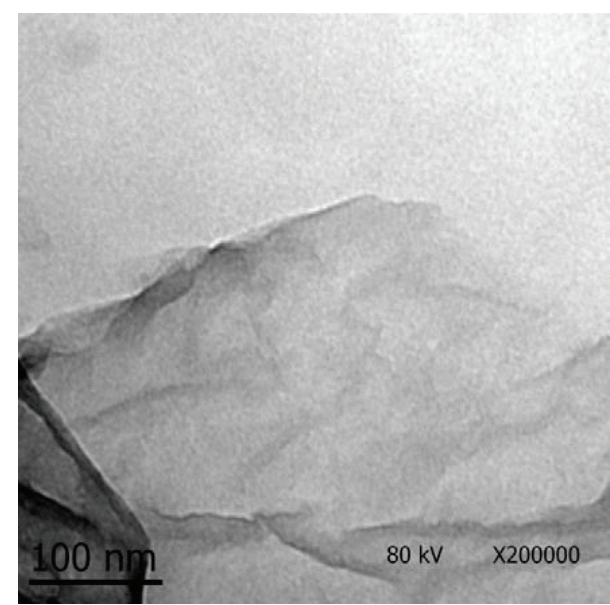

(b)

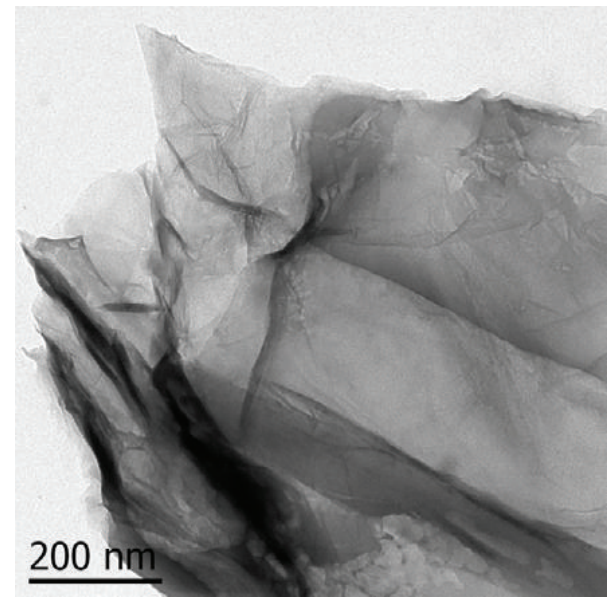

(d)

Figure 2: TEM images of (a)-(b) graphene oxide (GO) and (c)-(d) reduced graphene (RGO).

different sizes of both nanomaterials. Graphene oxide shows a wrinkled surface which provides stability and prevents collapse back to a graphitic structure [16]. Reduced graphene presents a similar morphology, like a thin extended film with wrinkled and rough surface. Both planar sheets offer more contact with the epoxy resin.

Table 1 shows the elemental analysis for graphene oxide and reduced graphene pure samples; the results correspond to the mean value of weight $\%$ and atomic $\%$ of four zones of the samples explored for EDS. The results show that graphene oxide contains $27.61 \mathrm{wt} \%$ of oxygen while reduced graphene only $16.38 \mathrm{wt} \%$, and then the ratio GO/RGO is 1.68 . The amount of carbon and oxygen was analyzed in order to estimate the proportion of oxygen presents in each one of the samples.

Figure 3 shows dispersion tests, of graphene oxide and reduced graphene oxide in water, DGEBA, and hexane. GO shows good dispersion in water (a) due to its polar oxygen functional groups that render it hydrophilic; however, it formed some precipitated after $24 \mathrm{~h}$. GO is less soluble in DGEBA (c); this resin is a non-water-soluble polymer and then its dispersion diminished and precipitated after $24 \mathrm{~h}$.
TABLE 1: Elemental analysis for graphene oxide (GO) and reduced graphene (RGO) of pure samples.

\begin{tabular}{lccc}
\hline Sample & Element & $\begin{array}{c}\text { Weight mean } \\
(\%)\end{array}$ & $\begin{array}{c}\text { Atomic mean } \\
(\%)\end{array}$ \\
\hline $\begin{array}{l}\text { Graphene oxide } \\
\text { (GO) }\end{array}$ & C K & 71.48 & 77.19 \\
\hline Reduced graphene & C K & 27.61 & 22.43 \\
(RGO) & O K & 83.28 & 87.03 \\
\hline
\end{tabular}

GO in hexane (e) started to precipitate few seconds after being retired of ultrasonic bath due to its incompatibility with the medium. On the other hand, reduced graphene oxide dispersed in water (b) precipitated after $1 \mathrm{~h}$. This nanomaterial became less hydrophilic as a result of oxygen removal. The increase in the hydrophobicity of the material caused less polar functionality on the surface of the nanosheets and increasing incompatibility with the aqueous medium $[13,17]$. This is corroborated when RGO is dispersed in DGEBA (d), 


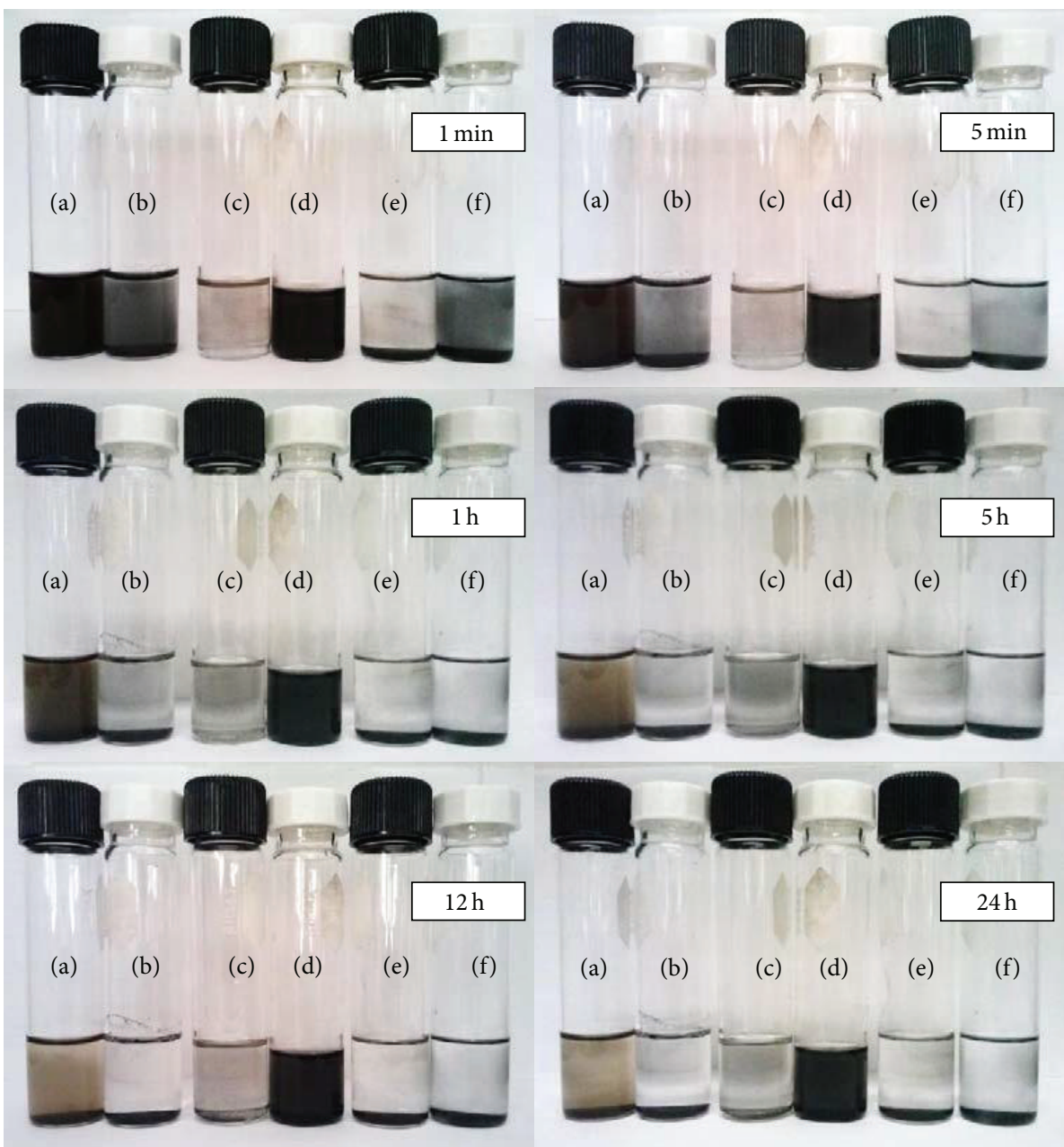

Figure 3: Samples of GO dissolved in water (a), DGEBA (c), and hexane (e) and RGO dissolved in water (b), DGEBA (d), and hexane (f) at different time lapses.

forming stable dispersion during $24 \mathrm{~h}$ (the most black dispersion). The nanosheets formed a homogeneous dispersion due to the hydrophobic character of both components making them more compatible. Reduced graphene in hexane (f) stayed dispersed some minutes and after being precipitated, however, it presented a better dispersion than GO.

3.2. Tensile Tests. The mechanical properties evaluated through tensile tests are shown in Figure 4. Stress-strain curves were obtained for all nanocomposites prepared in this work. We can observe an important improvement on tensile strength, elongation at break, and tensile toughness as a result of reinforcing with both nanofillers and using the different combinations of graphene oxide and reduced graphene. It is observed that at $0.4 \mathrm{wt} \%$ we obtain the best performance of all nanocomposites.

More details about tensile strength results are shown in Figure 5. Neat epoxy exhibits a tensile strength value of $31 \mathrm{MPa}$, and almost all nanocomposites show a better performance. Nanocomposites reinforced with only graphene oxide or reduced graphene at all loads present not high increments, but when both nanomaterials are combined, up to $52 \%$ enhancement is reached. The ultimate tensile strength was obtained with the ratio $0.25: 0.75 \mathrm{GO}: \mathrm{RGO}$ at $0.4 \mathrm{wt} \%$.

Figure 6 shows modulus of elasticity for all nanocomposites and pure epoxy. The major values are found at $0.4 \mathrm{wt} \%$ again and the combination $0.25: 0.75$ provides one of them, with $\sim 20 \%$ greater than the epoxy matrix. However, at least one combination shows similar results to those obtained with only graphene oxide or reduced graphene.

Another important parameter is the tensile toughness, which refers to the energy adsorbed by a material prior to fracturing. Figure 7 shows results of tensile toughness for all nanocomposites and neat epoxy. We found that with the ratio $0.25: 0.75 \mathrm{GO}: \mathrm{RGO}$ nanocomposites present outstanding performance at almost all loads of nanofillers. At $0.1 \mathrm{wt} \%$, performance is similar to using only reduced graphene (ratio $0: 1 \mathrm{GO}: \mathrm{RGO}$ ). Nevertheless, superior values of nanocomposites were obtained at $0.4,0.7$, and $1.0 \mathrm{wt} \%$ and the ultimate tensile toughness was achieved at $0.4 \mathrm{wt} \%$ showing $\sim 152 \%$ higher than neat epoxy. According to EDS results, graphene oxide contains $27.61 \mathrm{wt} \%$ of oxygen and reduced graphene $16.38 \mathrm{wt} \%$; then one gram of nanofiller 


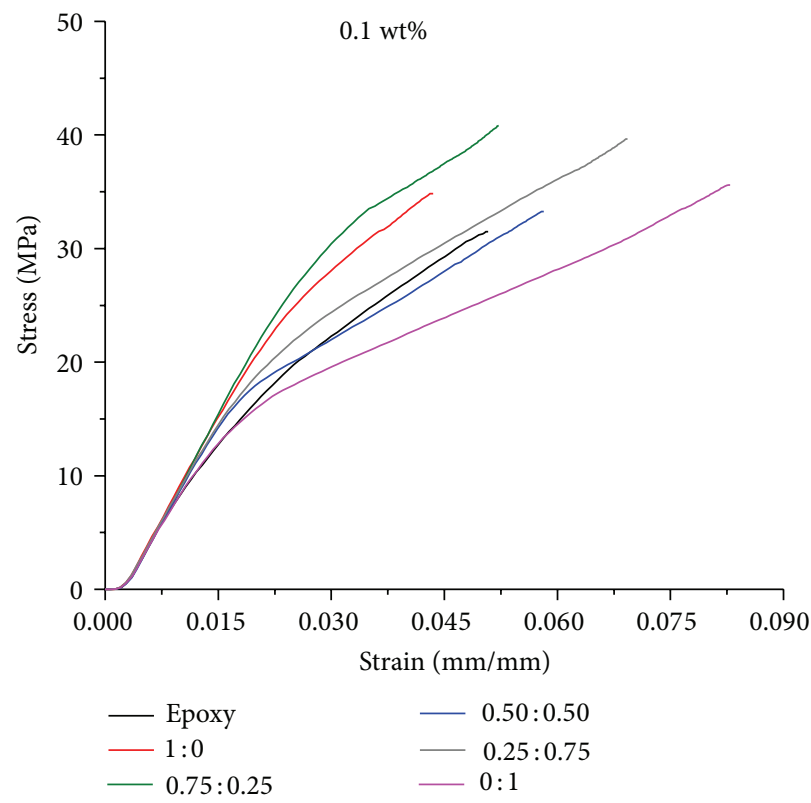

(a)

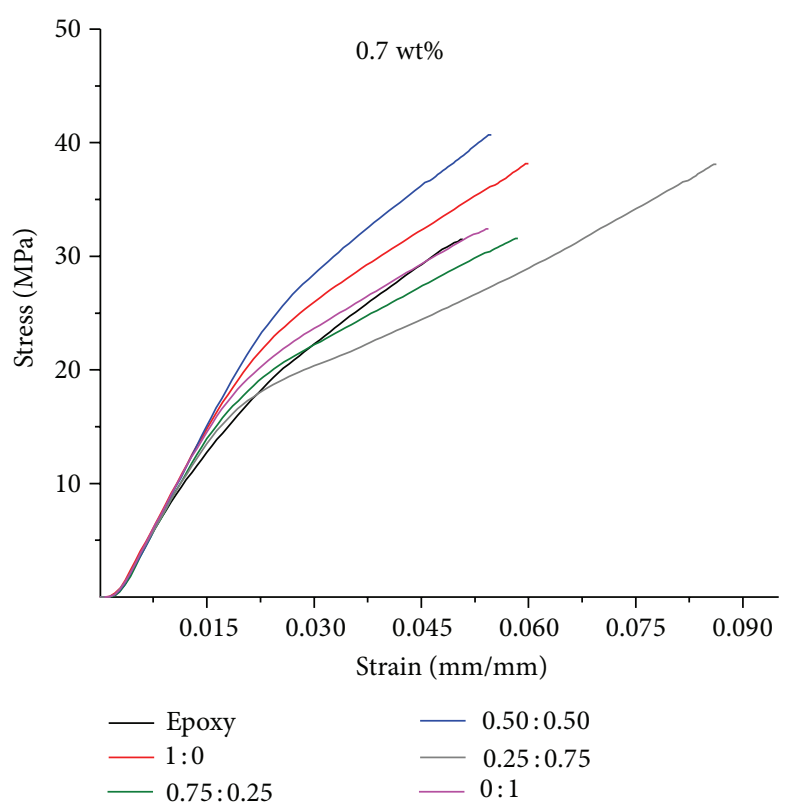

(c)

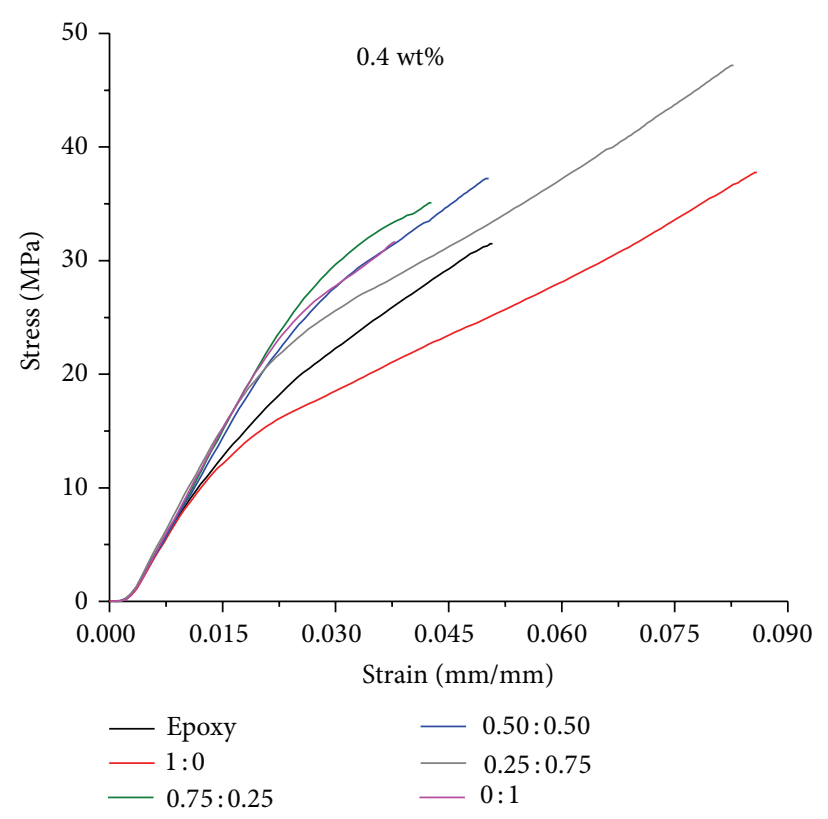

(b)

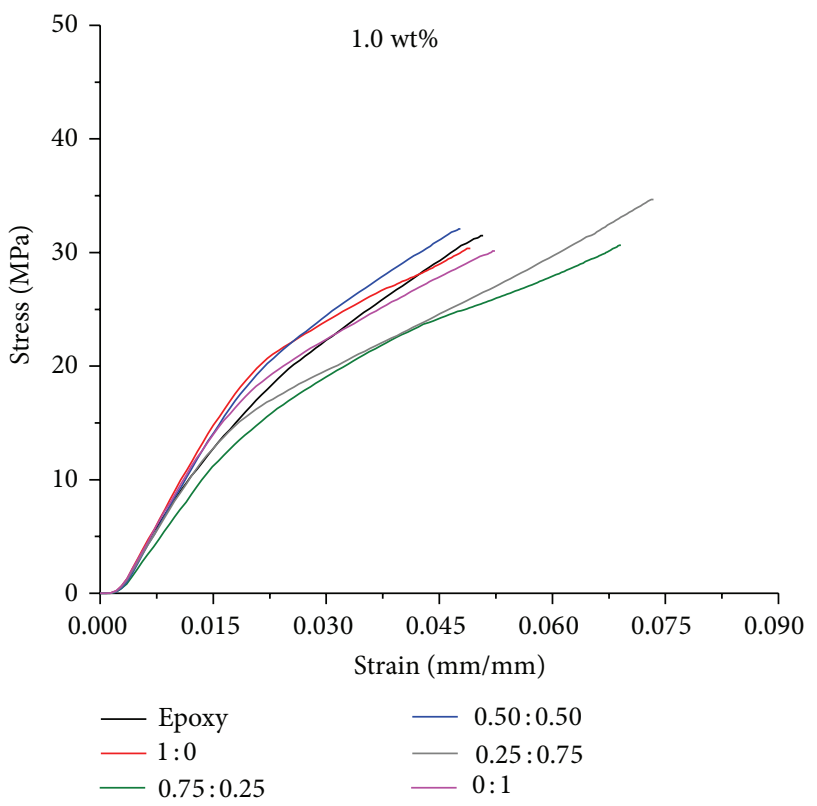

(d)

FIGURE 4: Stress-strain curves for nanocomposites at different loads and combinations.

of the mixture GO: RGO for $0.25: 0.75$ provides $0.1918 \mathrm{~g}$ of oxygen. The oxygen present in the mixture is susceptible to react and form interactions with the epoxy resin.

The improved mechanical properties presented can be explained in terms of amount of functionalized groups and compatibility with the matrix. It has been reported that oxygen groups over oxidized carbon materials can react with curing agent and form chemical bonds, which results in more interaction between nanofiller and matrix $[18,19]$. However, carboxylic groups found on the surface of GO can react with EDA leaving excess epoxy polymer without cross-linking [20]. Some authors [21-23] point out that the stoichiometric ratio of epoxy and amine groups is altered by the amount of oxygen groups, in this case over nanosheets. It is also known that graphene oxide has a hydrophilic character, which makes it less soluble in epoxy resin. On the other hand, reduced graphene recovers its hydrophobic character, making easier its dispersion in this matrix, but RGO does not have enough groups to bind chemically to the polymer. A lesser amount of oxygen groups present in the total reinforcement increases solubility, and yet there would be enough of them to bind to the matrix. This work has shown that a reduced amount of oxygen groups $(0.1918 \mathrm{~g})$ is enough to improve performance on nanocomposites. These results reveal that 

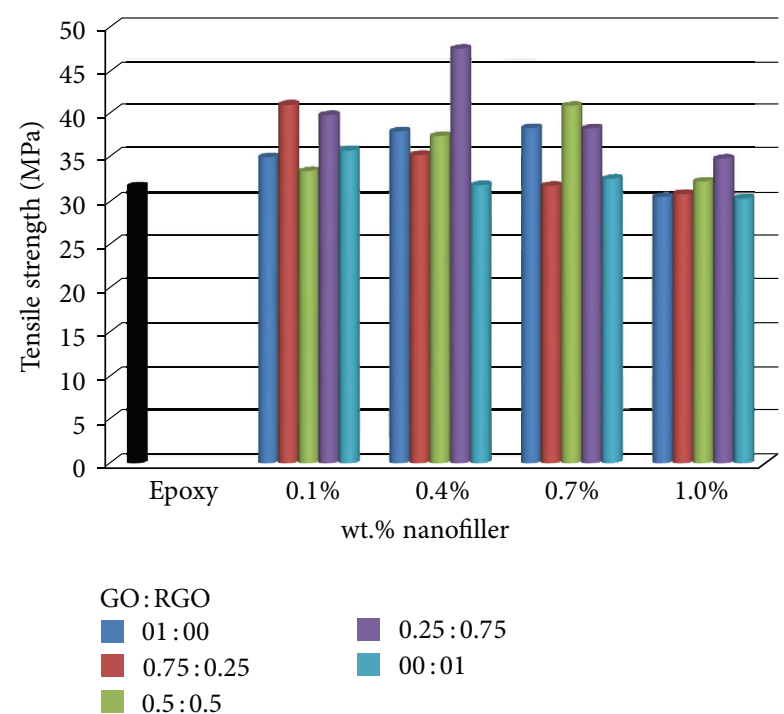

FIGURE 5: Tensile strength of nanocomposites reinforced with graphene oxide (GO), reduced graphene (RGO), and different combinations of both nanofillers.
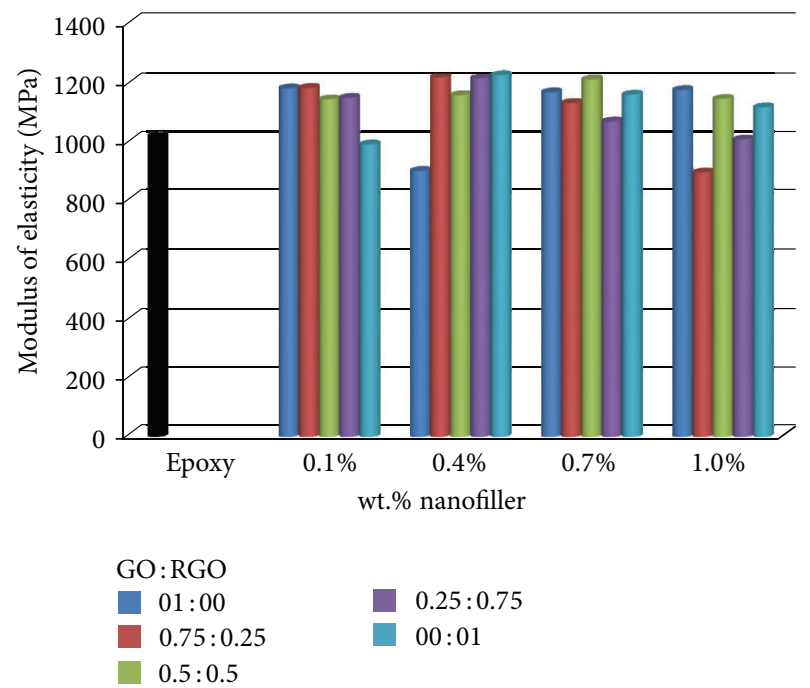

FIGURE 6: Modulus of elasticity of nanocomposites reinforced with nanomaterials.

employing only graphene oxide or reduced graphene is less effective; therefore, we find that there are greater possibilities to exploit the outstanding properties of graphene.

\section{Conclusions}

In this work, we found that the best performance in nanocomposites was obtained using nanofillers with characteristics between these two conditions of nanomaterials, oxidized and reduced. It was observed that with ratio $0.25: 0.75$ of GO: RGO the mechanical properties of nanocomposites were increased, and at $0.4 \mathrm{wt} \%$ and the same ratio, the ultimate performance was obtained. At this ratio $(0.25: 0.75$ of $\mathrm{GO}: \mathrm{RGO}$ ), the quantity of oxygen necessary to form
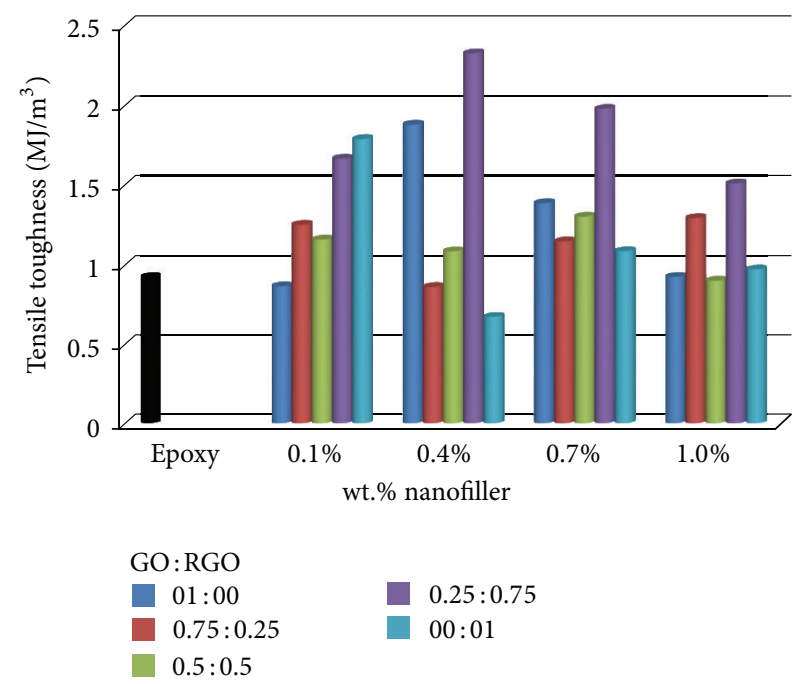

FIGURE 7: Tensile toughness of samples reinforced with nanomaterials.

chemical bonds was $0.1918 \mathrm{~g}$ per gram of mixture and the contribution of reduced graphene was essential to increase the compatibility with the matrix suggesting a synergic effect. The mixing GO: RGO purpose is an easy, quick, and cheap way to obtain nanocomposites with superior properties, since no additional chemical process is necessary.

\section{Conflict of Interests}

The authors declare that there is no conflict of interests regarding the publication of this paper.

\section{References}

[1] K. S. Novoselov, A. K. Geim, S. V. Morozov et al., "Electric field in atomically thin carbon films," Science, vol. 306, no. 5696, pp. 666-669, 2004.

[2] H. Kim, A. A. Abdala, and C. W. Macosko, "Graphene: electronic and photonic properties and devices," Macromolecules, vol. 10, no. 11, pp. 4285-4294, 2010.

[3] R. Verdejo, M. M. Bernal, L. J. Romasanta, and M. A. LopezManchado, "Graphene filled polymer nanocomposites," Journal of Materials Chemistry, vol. 21, no. 10, pp. 3301-3310, 2011.

[4] V. Singh, D. Joung, L. Zhai, S. Das, S. I. Khondaker, and S. Seal, "Graphene based materials: past, present and future," Progress in Materials Science, vol. 56, no. 8, pp. 1178-1271, 2011.

[5] R. J. Young and I. A. Kinloch, Graphene Composites, Wiley Encyclopedia of Composites, Wiley, New York, NY, USA, 2011.

[6] D. R. Cooper, B. D’Anjou, N. Ghattamaneni et al., "Experimental review of graphene," Condensed Matter Physics, vol. 2012, Article ID 501686, 56 pages, 2012.

[7] P. Song, L. Liu, S. Fu et al., "Striking multiple synergies created by combining reduced graphene oxides and carbon nanotubes for polymer nanocomposites," Nanotechnology, vol. 24, pp. 1-8, 2013.

[8] S. Chatterjee, F. Nafezarefi, N. H. Tai, L. Schlagenhauf, F. A. Nüesch, and B. T. T. Chu, "Size and synergy effects of nanofiller hybrids including graphene nanoplatelets and carbon 
nanotubes in mechanical properties of epoxy composites," Carbon, vol. 50, pp. 5380-5386, 2012.

[9] R. J. Young, I. A. Kinloch, L. Gong, and K. S. Novoselov, “The mechanics of graphene nanocomposites: a review," Composites Science and Technology, vol. 72, no. 12, pp. 1459-1476, 2012.

[10] B. Z. Jang and A. Zhamu, "Processing of nanographene platelets (NGPs) and NGP nanocomposites: a review," Journal of Materials Science, vol. 43, no. 15, pp. 5092-5101, 2008.

[11] D. R. Bortz, E. G. Heras, and I. Martin-Gullon, "Impressive fatigue life and fracture toughness improvements in graphene oxide/epoxy composites," Macromolecules, vol. 45, no. 1, pp. 238-245, 2012.

[12] Z. Spitalsky, G. Tsoukleri, D. Tasis, C. Krontiras, S. N. Georga, and C. Galiotis, "High volume fraction carbon nanotube-epoxy composites," Nanotechnology, vol. 20, no. 40, Article ID 405702, pp. 778-783, 2009.

[13] S. Mao, H. Pu, and J. Chen, "Graphene oxide and its reduction: modeling and experimental progress," RSC Advances, vol. 2, no. 7, pp. 2643-2662, 2012.

[14] M. J. Fernández-Merino, L. Guardia, J. I. Paredes et al., "Vitamin $\mathrm{C}$ is an ideal substitute for hydrazine in the reduction of graphene oxide suspensions," Journal of Physical Chemistry C, vol. 114, no. 14, pp. 6426-6432, 2010.

[15] M. Acik, G. Lee, C. Mattevi et al., "The role of oxygen during thermal reduction of graphene oxide studied by infrared absorption spectroscopy," Journal of Physical Chemistry C, vol. 115, no. 40, pp. 19761-19781, 2011.

[16] H. Wu, J. Wang, X. Kang et al., "Glucose biosensor based on immobilization of glucose oxidase in platinum nanoparticles/graphene/chitosan nanocomposite film," Talanta, vol. 80, no. 1, pp. 403-406, 2009.

[17] D. R. Dreyer, S. Park, C. W. Bielawski, and R. S. Ruoff, "The chemistry of graphene oxide," Chemical Society Reviews, vol. 39, no. 1, pp. 228-240, 2010.

[18] C. Velasco-Santos, A. L. Martínez-Hernández, and V. M. Castaño, "Carbon nanotube-polymer nanocomposites principles and applications," in Focus on Nanotube Research, D. A. Martin, Ed., pp. 97-126, Nova Science Publishers, Huntington, NY, USA, 2006.

[19] V. Datsyuk, M. Kalyva, K. Papagelis et al., "Chemical oxidation of multiwalled carbon nanotubes," Carbon, vol. 46, no. 6, pp. 833-840, 2008.

[20] L. Sun and H. Sue, "Chapter 10. Epoxy/Carbon nanotube nanocomposites," in Epoxy Polymers: New Materials and Innovations, J. P. Pascault and R. J. J. Williams, Eds., pp. 185-211, WILEY-VCH, Cambridge, UK, 2010.

[21] J. D. Fidelus, E. Wiesel, F. H. Gojny, K. Schulte, and H. D. Wagner, "Thermo-mechanical properties of randomly oriented carbon/epoxy nanocomposites," Composites A, vol. 36, no. 11, pp. 1555-1561, 2005.

[22] J. E. Ehlers, N. G. Rondan, L. K. Huynh, H. Pham, M. Marks, and T. N. Truong, "Theoretical study on mechanisms of the epoxy-amine curing reaction," Macromolecules, vol. 40, no. 12, pp. 4370-4377, 2007.

[23] Y. Zhang, A. A. Broekhuis, M. C. A. Stuart et al., "Cross-linking of multiwalled carbon nanotubes with polymeric amines," Macromolecules, vol. 41, no. 16, pp. 6141-6146, 2008. 

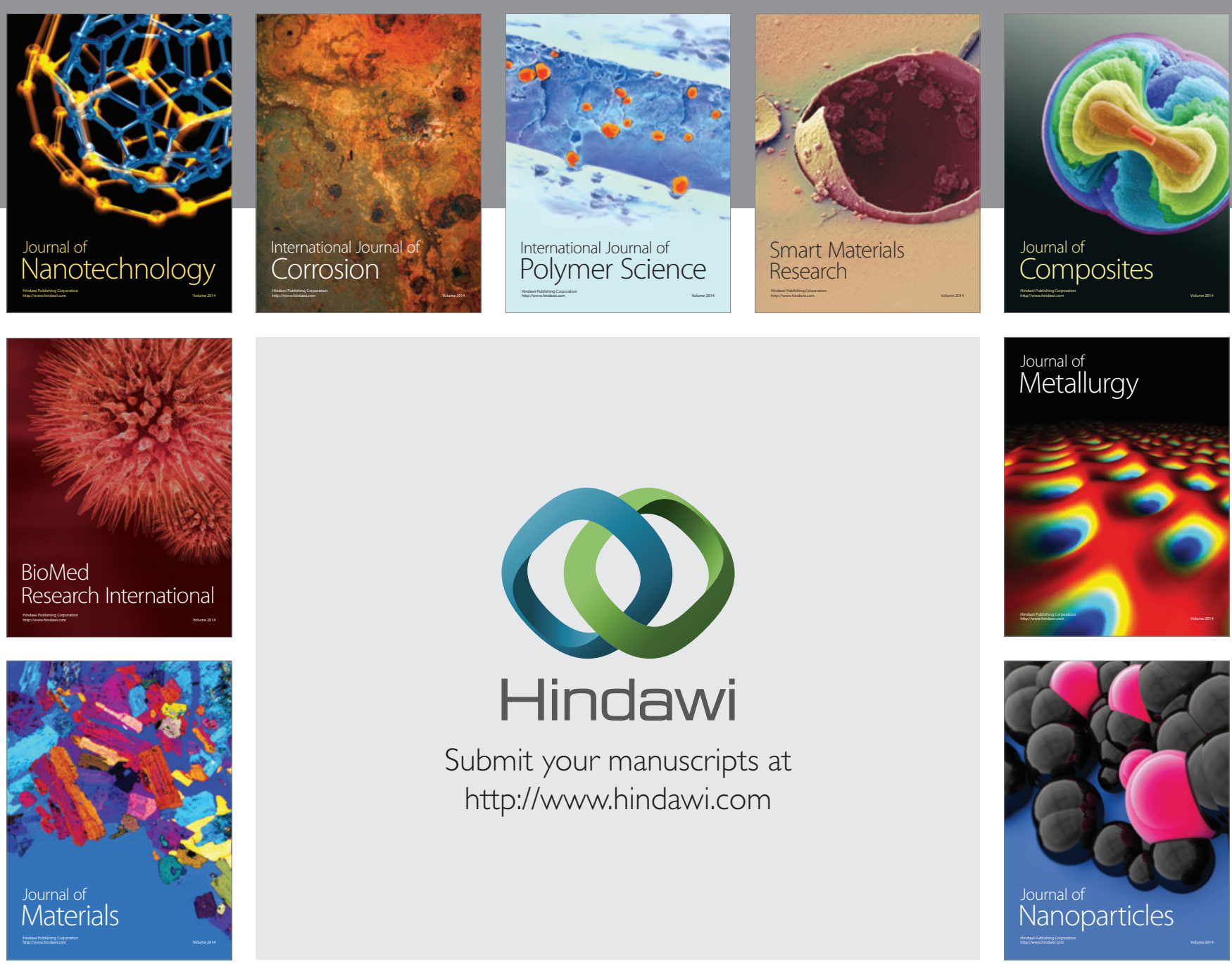

Submit your manuscripts at http://www.hindawi.com
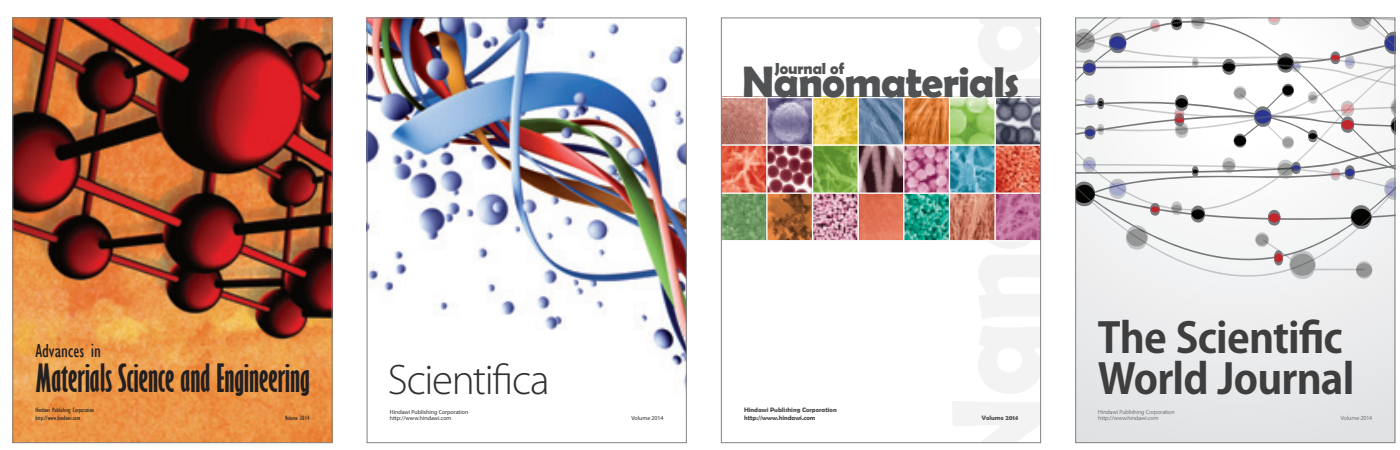

\section{The Scientific World Journal}
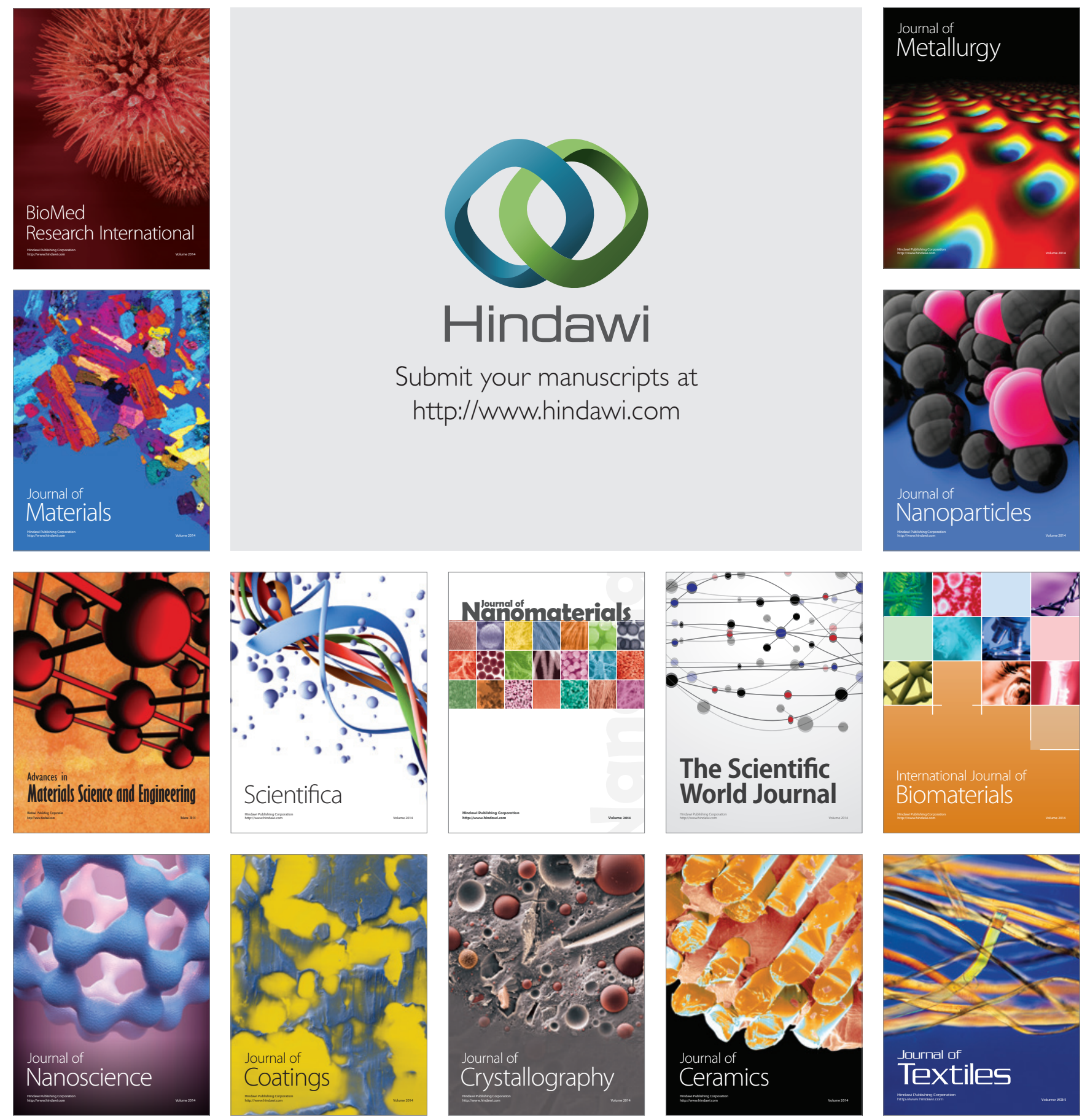